\title{
De l'analogie à l'anamorphose : visions du corps musical dans le drame shakespearien
}

\section{Pierre Iselin}

\section{(2) OpenEdition \\ 1 Journals}

\section{Édition électronique}

URL : http://journals.openedition.org/shakespeare/1200

DOI : 10.4000/shakespeare.1200

ISSN : 2271-6424

Éditeur

Société Française Shakespeare

\section{Édition imprimée}

Date de publication : 1 novembre 1991

Pagination : 153-174

Référence électronique

Pierre Iselin, « De l'analogie à l'anamorphose : visions du corps musical dans le drame shakespearien », Actes des congrès de la Société française Shakespeare [En ligne], 9| 1991, mis en ligne le 01 janvier 2007, consulté le 02 mai 2019. URL : http://journals.openedition.org/shakespeare/1200 ; DOI : 10.4000/shakespeare.1200 


\section{SHAKESPEARE \\ ET LE CORPS A LA RENAISSANCE}

Société Française Shakespeare

Actes du Congrès 1990

sous la direction

de

M.T. JONES-DAVIES

PARIS

LES BELLES LETTRES

1991 
Tous droits de traduction, de reproduction et d'adaptation réservés pour tous les pays.

(C) 1991 Société d'édition Les Belles Lettres, 95 bd Raspail 75006 Paris

ISBN $2.25169117-\mathrm{X}$ 


\section{De l'analogie à l'anamorphose : visions du corps musical dans le drame shakespearien}

Jour sans doute prédestiné à la plaisanterie et à la dérision, $c^{\prime}$ est le $1^{\text {er }}$ avril (1710) qu'Addison publiait dans The Tatler une sorte d'autobiographie, où, s'incarnant successivement en cinq instruments, il prenait donc les traits, c'est-à-dire l'ethos, du tambour, de la trompette, du flageolet-tambourin, du luth, et en fin de parcours, de la cornemuse :

For my part, I must confess, I was a drum for many years; nay, and a very noisy one, till having polished myself a little in good company, I threw as much of the trumpet into my conversation as was possible for a man of an impetuous temper, by which mixture of different musics, I look upon myself, during the course of many years, to have resembled a tabor and pipe. I have since very much endeavoured at the sweetness of the lute; but in spite of all my resolutions, I must confess with great confusion, that I find myself daily degenerating into a bagpipe. ${ }^{1}$

C'est bien l'instrumentarium fantastique et symbolique de la Renaissance qui défile ainsi mécaniquement dans cette rhétorique de métaphores mortes, codifiées à l'extrême. Un Pythagore d'opérette semble y mettre en scène une sorte de palingénésie instrumentale, où les ethoi instrumentaux parfaitement identifiables jettent des passerelles devenues trop sûres et fréquentées entre corps humain 
et instrument de musique. Ces similitudes, perçues sur le mode de la dérision, semblent vouloir faire écho au discours élisabéthain, tel celui de Jaques sur les âges de la vie, où la dégénérescence physique métamorphose la voix virile en instruments et sons aigus :

his big manly voice,

Tuming again toward childish treble, pipes

And whistles in his sound. ${ }^{2}$

On peut penser à des dizaines d'autres allusions ou analogies, comme à cette description, brossée par Rabelais, des pauvres "vérolés et goutteux" dont les «dentz leurs tressailloyent comme font les marchettes [= touches] d'un clavier d'orgues ou d'espinette quant on joue dessus» ${ }^{3}$, ou à cette anatomie musicale de Quaresmeprenant : «les orteilz avoit comme une espinette orguanisée ... Le nombril, comme une vielle ... Le perinæum, comme un flageollet ... Les spondyles, comme une cornemuse ... La poictrine, comme un jeu de réguales. Les mammelles comme un cornet à bouquin. Les aisselles, comme un eschiquier ${ }^{4}$... Les paulpières, comme un rebec ... La langue, comme une harpe»5. Même analogie, même scénario, même effet comique peut-être. Pourtant la dérision ne fonctionne pas selon le même modèle : ce que le siècle des lumières brocarde sous la plume d'Addison, c'est le mode analogique lui-même autant que l'idiome musical par lequel il est si fréquemment signifié. Il est vrai, pour reprendre la conclusion de John Hollander, que la musique même ne remplit pas la même fonction: elle n'est plus, dès le début du XVII siècle, a fortiori au XVIII', qu'un modèle thétorique - au même titre que les images surannées du Tatler - dont l'efficacité réside dans sa capacité d'émouvoir, plutôt que dans celle, à la fois magique et heuristique, de donner un modèle d'ordre universel ${ }^{6}$, selon un système de similitudes déjà passablement exploré?.

Le rapport du corps à l'instrument et sa réciproque reposent en effet sur un code esthétique, philosophique et somme toute idéologique, en pleine mutation et en pleine discussion. Les traités sur la musique élaborent tradition et idées reçues en une rhétorique stéréotypée, dont on trouve des traces évidentes dans la dramaturgie contemporaine. C'est cet intertexte qui constituera le champ de cette étude sur le fantastique musical. 


\section{VIXTYS. EMBLEYIS}

Alliances.

A>OSIXOPHZ

Sur l'Alliance des Fraliens.

A BOSIROPHE.

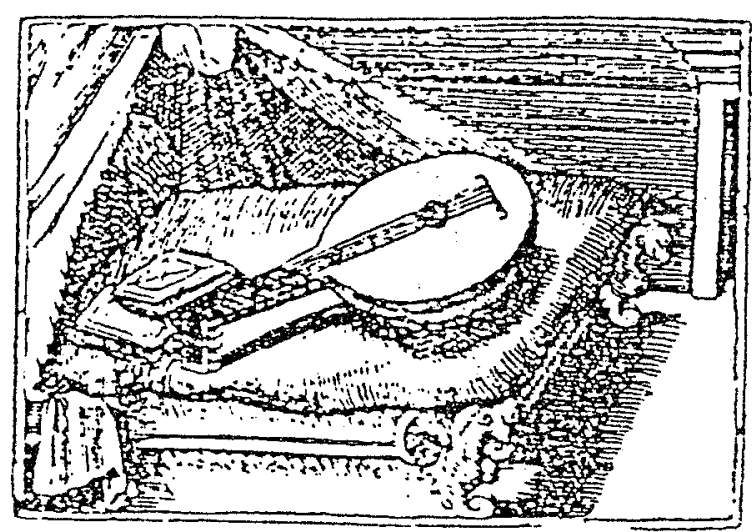

1. Alclat, Toutes Ies Emblemes, Lyon, 1558, , p. 30 .

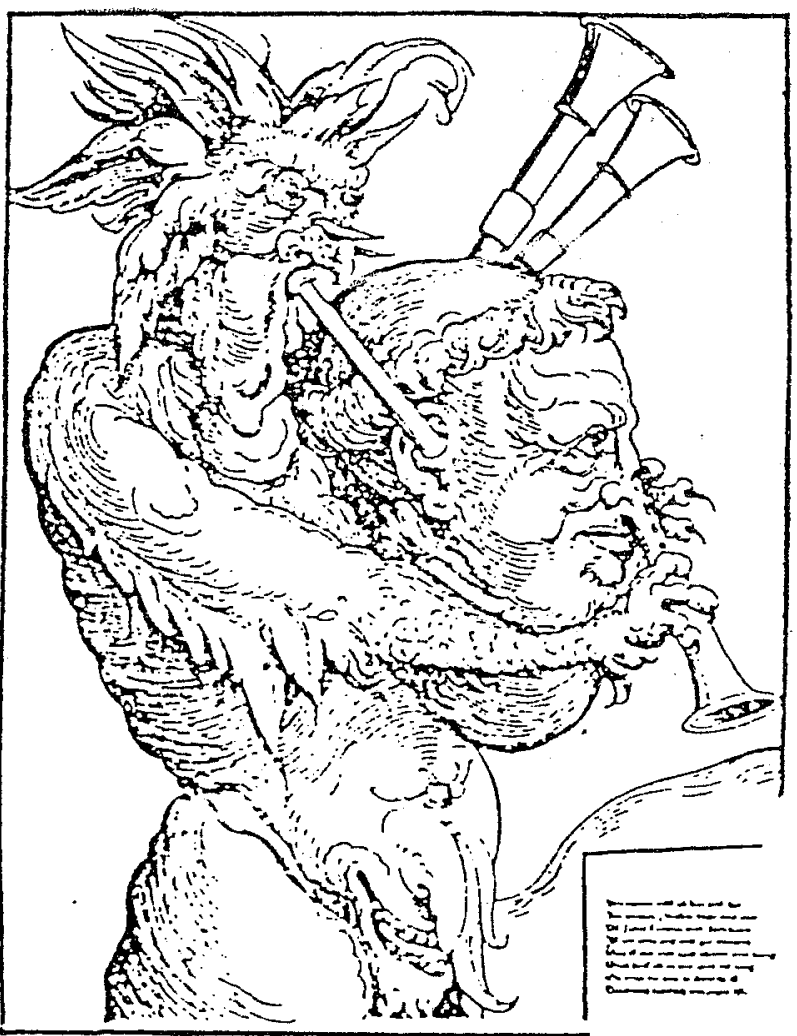

3. Gravure sur bols, anon. Allemagne, env.1535. Document de la propagande ant1-papiste. 


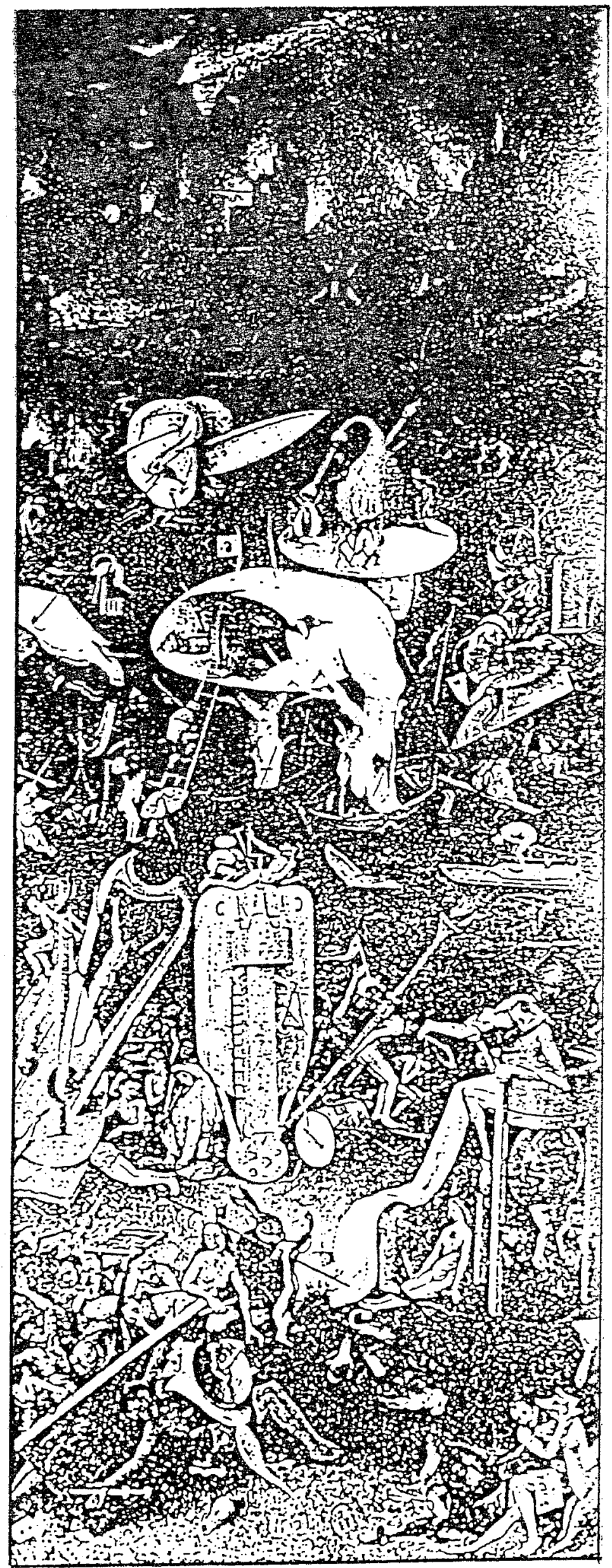

2. Jérôme Bosch. L'Enfer. 
Le premier principe de l'analogie corps-instrument est à chercher dans l'organisation musicale de toute création, puisque l'outil mathématique qu'est le rapport (la proportion) peut indifféremment s'écrire en données chiffrées ou harmoniques, comme de nos jours l'espace peut s'exprimer en temps, et réciproquement. La construction des intervalles (rapports de nombres entiers) de la gamme, et donc l'instrument qui leur donne voix est ainsi analogue à cet instrument qu'est le corps humain en ce que toutes ses proportions s'expriment dans la même unité de mesure. Par exemple, la distance entre le sommet du crâne et le nez «résonne (risuona) avec la distance du nez au menton dans une proportion triple [rapport 3/1]; et ladite distance du nez au menton et celle du menton à la rencontre des clavicules résonne dans une proportion double [2/1] qui fait le diapason [1'octave] ${ }^{8}$.

La connaissance des rapports harmoniques passe donc par la musique, et l'architecte applique ainsi les consonances musicales du corps humain au corps de bâtiment ${ }^{9}$, l'expérience spatiale étant subsumée par l'expérience acoustique; les proportions géométriques du corps humain, que Léonard de Vinci et Francisco di Giorgi redécouvrent dans leurs études vitruviennes, établissent la norme architecturale, exactissime harmonica, faite de rapports simples analogues à ceux des intervalles consonants (diapason, diapente, diatessaron ...). Ainsi le mythe d'Amphion trouve-t-il dans les traités d'architecture du XVIe siècle une lecture à la fois très littérale et rationalisée.

C'est en pensant à cette analogie (qu'il élargit à un champ beaucoup plus vaste), que l'auteur anonyme ${ }^{10}$ d'un Eloge de la Musique, The Praise of Musicke (Oxford, 1586), forge en anglais le terme de "symbolisme" (simbolisme ${ }^{11}$ ), qu'il définit selon des termes quasi architecturaux :

To prove this looke upon the frame, and workmanship Agrippa of the whole worlde, whetherthere be not above, an Musica harmony between the spheares, beneath a simbolisme Mundana between the elements. Looke upon a man, whom the Cor. Philosophers termed a litle world, whether the parts accord not one to the other by consent and unity. ${ }^{12}$

Ce "symbolisme", que l'on peut paraphraser en "harmonies", "similitudes" ou "rapports proportionnels" fonde donc cette "musique humaine" (musica humana) sur un système d'analogies 
et de congruences entre partie et tout, et, au-delà du constat strictement anthropomorphique, d'une totale correspondance entre le corps et son environnement terrestre et céleste. Dans cette perspective, la comparaison du corps humain à un instrument tient davantage de l'élucidation que de l'ornement. Cinquante ans après la publication de Praise, Charles Butler, dans The Principles of Musicke, formule toujours cette idée reçue dans des termes très proches :

For they prais God upon the wel-tuned Cymbals, upon the Harp, and Psalteri of ten strings, is a note and signification that there is such a concent betweene the partes of the body, as there is among the strings. ${ }^{13}$

L'isomorphisme entre le corps et linstrument à cordes est ici encore exprimé en termes abstraits, quasi mathématiques (concent), où l'on reconnaît l'empreinte des Pythagoriciens et néo-Platoniciens, et du système de correspondances hermétiques élaboré par Ficin, Cornelius Agrippa, Fludd, John Dee, ou plus tard Kircher ${ }^{14}$. La musique, et donc l'instrument qui l'exprime, est un moyen d'investiguer l'ensemble de la création où, selon John Dee, tout est analogue : «The whole world is, as it were, a lyre ... and man too, for all is analogus» ${ }^{15}$. L'harmonie du monde, la relation de lâme au corps et au monde, les relations des hommes entre eux, le fonctionnement des organes ont donc en commun un mode d'écriture, dont les hiéroglyphes sont convertibles en langage musical.

La statut ambigu qu'assume la musique, à la fois modèle d'une harmonie globale et reflet de celle-ci, tient au double statut que ses théoriciens postulent : elle est reflet imparfait dans sa dimension acoustique (musica practica), modèle heuristique dans sa dimension conceptuelle (musica speculativa).

Dans cette perspective quasi-impérialiste du concept musical, ce n'est pas seulement le corps humain, ce ne sont pas seulement les corps célestes, qui obéissent aux proportions de l'harmonie. Le corps social est lui aussi un macro-instrument de musique, accordé par le monarque ou les puissances supérieures (powers above ${ }^{16}$ ) selon le cas. Le monocorde de Fludd ${ }^{17}$ ou l'orgue de Kircher $^{18}$, représentations emblémiques du monde, ont un équivalent shakespearien dans ce tonnerre au langage prodigieux décrit comme un terrible tuyau d'orgue : 


\section{Alonso. $\quad \mathrm{O}$, it is monstrous \\ Methought the billows spoke and told me of it; \\ The winds did sing it to me; and the thunder, That deep and dreadful organ-pipe, pronounc'd The name of Prosper : it did bass my trespass. ${ }^{19}$}

Version acoustique et thêâtrale de l'œil de Dieu pourchassant Caïn, l'instrument, divin ou magique, a la fonction du deus ex machina, rappelle au coupable son fratricide, et s'intègre dans l'espace sonore magique de lìle régi par Ariel, dont le nom même connote les pouvoirs aériens, donc musicaux ${ }^{20}$. L'image du monde-instrument se traduit au niveau de l'organisation sociale du Body Politick par le même type de conceit : le corps politique, malade, en rébellion ou en guerre civile, est régulièrement assimilé à un instrument désaccordé, et le monarque a donc la charge difficile de tenir acordées toutes les cordes de l'instrument. Les livres d'emblèmes de la Renaissance, comme celui d'Alciat ${ }^{21}$, représentant l'alliance des Italiens par la «comparaison d'un Luc [= luth] accordé ou discors» (illustration 1); l'inscription "fides" 22 (dans l'édition latine originale) joue sur les mots (confiance /yre), comme les images jouent sur l'analogie conceptuelle musique/ordre. John Case, quant à lui, consacre dans son Apologia Musices (1588), un chapitre entier aux rapports de la politique et de la musique, et un livre presque entier (VIII) dans son autre traité. Sphoerc Civitatis.

C'est par le truchement du côps humain qu'il établit cette relation : si l'ordre dans la cité n'est pas calqué sur l'harmonie du musicien, alors s'ensuit la confusion sociale :

[...] ut dissolutis nervis corpus in paralysin et tremorem partium : ita civitas dissonis membris convulsa in horrorem et confusionem ipsorum civium pericolose cadit. ${ }^{23}$

Selon le symbolisme défini et illustré plus haut, Richard III traduit sa propre difformité physique en dissonance :

I, that an curtail'd of this fair proportion, ${ }^{24}$

et, faute de pouvoir jouir des passe-temps frivoles et lascifs qu'offre un temps de paix, il se propose d'improviser un déchant (c'està-dire un contrepoint) sur son infirmité :

Why, $I$, in this weak piping time of peace, 
Have no delight to pass away the time, Uniess to see my shadow in the sun And descant on mine own deformity. ${ }^{25}$

On voit comment Shakespeare, selon une pratique récurrente, intègre en les inversant dans sa dramaturgie des éléments hérités de la théorie, et des iđáes reçues sur la musique.

L'harmonie céleste, sociale, conjugale ou personnelle n'est perçue dans le drame qu'a contrario, par une mise en scène des dissonances. Sans faire nécessairement siens les concepts symbolistes de "musique" et de "tempête" que G. Wilson Knight élabore en système polaire, il est néanmoins inhérent au drame de se nourrir de tensions, et donc de ne réserver à la consonance et à lhamonie qu' une place, certes essentielle, mais marginale, soit dans le tableau d'ouverture, soit dans un cheur ou une danse finale, symbole dramatique de la réconciliation et de la Tête.

La dérision de I'harmonie, si persistante dans le texte shakespearien, peut donc sinscrire dans la poétique même de l'ouvre dramatique, comme l'un de ses ressorts fondamentaux. Elle constitue 6 galement un artifice comique efficace lorsque le dramaturge provoque l'écart maximal, la distorsion/dissonance la plus significative entre un agent avéré de la subversion et les concepts les plus élaborés de la musique spéculative.

Ainsi le bourfon dOthello, à lacte $\Pi$, scène 1, se livre-t-il à une mise en scène burlesque, où il réclame, au nom du général, une musique - sous-entendu, celle des sphères - réputée inaudible aux mortels. La requête est d'autant plus saugrenue que cette harmonie inaudible ne pourra désormais plus restaurer l'harmonie conjugale savamment désaccordée par lago:

Clown. If you have any music that may not be heard, to 't again, but, as they say, to hear music, the general does not greatly care. ${ }^{26}$

C'est cette harmonie-là que revendique également Gosson, dans cette apologie du silence qu'est à bien des égards The Scholle of Abuse (1579):

Pythagoras bequeathes them a Clooke bagge, and condemnes them for fooles, that judge Musicke by sounde and eare. If you will bee good schoolars, and profite well in the Arte of Musicke, shutte your Fidels in their cases, and looke up to heaven: the order of the $S p h e r e s$, the unfallible motion of the Planets, the juste course of 
the yeere, and varieties of seasons, the concorde of the Elementes and their qualyties, Fyre, Water, Ayre, Earth, Heate, Colde, Moysture and Drought concurring together to the constitution of earthly bodies and sustenance of every creature. ${ }^{27}$

Eloge et critique de la musique ont une logique, et même un langage communs : la tripartition corps-âme-cosmos se reproduisant depuis Boèce dans la notion même de musique, les données perceptuelles brutes (musica instrumentalis) ne sont qu'un écho très imparfait - et dans l'épaisseur de ce "très" réside toute la différence entre un Gosson et un Case - mais un écho tout de même, de l'harmonie du monde (musica mundana). Médium privilégié de la connaissance, outil exploratoire de l'alchimiste, elle est, au carrefour de correspondances occultes, l'expression d'un "symbolisme entre les éléments", que la terminologie comme à dessein, vient confirmer. En effet, les représentations anthropomorphiques très fréquentes sur violes de gambes, cistres ${ }^{28}$ et rebecs, ne sont que l'aspect visuel d'une similitude que le lexique pousse beaucoup plus loin. Anatomie et organologie expoitent, semble-t-il par système, le même champ sémantique. Ne définit-on pas corps et instruments par les mêmes termes de "body", "neck", "shoulders", "back", "sides", "ribs", "belly", "cords", "organ", "pipe", "voice", "holes", ou même "virginal"? Ne parle-t-on pas en français d'instruments inanimés qui auraient une "âme" (soundpost) et des "ouïes" (holes)? Les formes suggestives de la viole de gambe ou du luth s'inscrivent dans cette constellation anthropomorphique, comme bien entendu la tenue même de l'instrument. C'est chez Marston que ce rapport est exprimé de la manière la plus explicite, quoique les termes de l'analogie y soient ironiquement inversés :

Sly. O cousin, come, you shall sit between my legs, here.

Sinklo. No, indeed, cousin, the audience then will take me for a viol da gamba, and think that you play upon me..$^{29}$

Et tout luthiste connaît bien la rumeur selon laquelle l'instrument, pour ne pas subir les variations de température qui sont fatales à son accord, doit être gardé au lit. Le roi des instruments se lamente même comme une compagne : la voix artificielle ${ }^{30}$ pleure son maître, ici Philip van Welder, luthiste du roi Henry VIII :

The string is broke, the lute dispossest

The hand is colde, the bodye in the grounde. 
The lowring lute lamenteth now therfore

Philips her frende that can her touche no more. ${ }^{31}$

Le fifre, quant à lui, s'il ne mérite pas les conceits réservés aux instruments nobles, est toutefois marqué du même sceau : par l'hypallage, Shylock le pourvoit d'une définition corporelle, tout à fait caractéristique : il a le cou tors (wry neck'd fiffe ${ }^{32}$ ).

On sait bien lissue tragique de la gestuelle risible associee aux instruments à vent: Minerve lance l'aulos maudit que Marsyas n'aurait jamais dû ramasser, eût-il un seul instant tenu à son épiderme! Alcibiade, comme Minerve et Shylock, récuse la disgrâce des mouvements, la position du corps et les mimiques grotesques qu'implique la pratique de la flûte, à laquelle il est rebelle :

Afterwards when he was put to schoole to learne, ... he disdained to learne to playe of the flute or recorder: saying, that it was no gentlemanly qualitie. For, sayed he, to playe on the vyoll with a sticke, doth not alter mans favour, nor disgraceth any gentleman: but otherwise, to play on the flute, his countenance altereth and changeth so ofte, that his familiar friends can scant knowe him. Moreover, the harpe or vyoll doth not let him that playeth on them, from speaking, or singing as he playeth: where he that playeth on the flute, holdeth his mouth so harde to it, that it taketh not only his wordes from him, but his voyce. ${ }^{33}$

L'instrument de musique peut en effet par le jeu sémantique autant que par le jeu scénique devenir instrument de torture. Etymologie, mythologie et dramaturgie nous présentent régulièrement des tableaux en anamorphose où l'instrument de l'harmonie en cache un autre, de destruction ${ }^{34}$ : Linus, le maître de musique d'Hercule, ne fut-il pas tué, se-lon Apollodore ${ }^{35}$, par son propre élève d'un coup de cithare?

Figure sacrificielle du mythe, le musicien martyr est aussi figure comique. La leçon de luth qu' Hortensio est censé donner à Katherina tourne au pugilat, et le luth devient pilori :

Bap. Why then, thou canst not break her to the lute?

Hor. Why no, for she hath broke the lute to me. ... she struck me on the head, And through the instrument my pate made way, And there I stood amazed for a while, As on a pillory, looking through the lute ... ${ }^{36}$ 
Dans la même lignée, Orphée déchiqueté par les Bacchantes, Arion jeté à la mer, Marsyas écorché vif, Demodocus ${ }^{37}$ assassiné par Egiste sont autant de musiciens martyrs, dont les pouvoirs inquiètent dans ce monde et dans l'autre. Les instruments portent aussi en eux-mêmes la mémoire du corps torturé : l'aulos inventé par Athéna veut imiter le cri de mort d'Euryale, l'une des trois Gorgones, décapitée par Persée ${ }^{38}$. C'est sous la forme d'un roseau, dont on fait l'anche, que la nymphe Syrinx se métamorphose pour fuir $\mathrm{Pan}^{39}$. La flûte ne sera toujours qu'un tibia, le cor et le comet à bouquin des cornes, la corde de luth du boyau de chat, l'archet du crin de cheval, et le premier instrument une carapace de tortue ${ }^{40}$. Et même par delà la mort, les vieilles sympathies ${ }^{41}$, comme les vieilles antipathies perdurent. Pierre Trichet rapporte dans son Traité des Instruments de Musique (ca. 1640) une "expérience" acoustique fréquemment décrite dans les traités ${ }^{42}$, la corde composée par moitié de boyau d'agneau et de boyau de loup ne peut être accordée, «l'antipathie entre ces deux animaux estant si grande que mesme après leur mort elle ne cesse de persévérer». Une fable analogue rend linstrument tendu de boyaux de serpents responsable de nombreux avortements ${ }^{43}$. Marston, dans What You Will évoque ce rapport de l'instrument à ses composantes organiques :

Now the musiciens

Hover with nimble sticks o'er squeaking crowds

Tickling the dried guts of a mewing cat.

Shakespeare va plus loin encore lorsque le luth de la séduction a pour cordes les tendons même d'Orphée :

For Orpheus' lute was strung with poets' sinews,

Whose golden touch could soften steel and stones, ${ }^{44}$

La figure d'un Orphée crucifié 45 apparaît ainsi dans l'instrument même du poète maudit, mais également en palimpseste, par dessus l'image de sa dislocation physique rapportée par la légende.

La harpe irlandaise tire également ses pouvoirs orphiques de sa composante organique : tendue de boyaux de lynx (lug en irlandais) elle hérite par homonymie des pouvoirs du dieu Lug $^{46}$. Peut-on voir dans cette "signature" à la Crollius ${ }^{47}$ l'etymologie d'un catgut qui n'a jamais réellement existé en lutherie? Pour Benedick, l'extase musicale se réduit non pas aux vertus du mode phrygien, mais à celle du boyau de mouton : 
Now, divine air! Now is his soul ravished!

Is it not strange that sheep's guts should

Hale souls out of men's bodies? ${ }^{48}$

Platon $^{49}$, Macrobe ${ }^{50}$, Hooker ${ }^{51}$, l'auteur anonyme de The Praise of Musicke, et tant d'autres sont à l'arrière-plan de cette conception quasi-anatomique de l'ex-stase. Les poètes de la Pléiade également, pour qui le ravissement a une définition semble-t-il standardisée : la musique «tire l'âme par l'oreille» ${ }^{52}$

A cette vision pseudo-béatifique de la séparation, de la distinction, si proche de celle de Lorenzo, s'oppose la vision infernale où le corps s'amalgame à l'instrument. Ces métamorphoses fantastiques ont sans doute trouvé leur expression plastique extrême dans le Jardin des Délices ou le Jugement Dernier de Jérôme Bosch (illustration 2): le luth y prend des allures de chevalet, la harpe y devient un motif de crucifixion, des monstres antédiluviens jouent sur un nez hypertrophié qui s'anastomose en hautbois; chalémie, buisine et cornemuse, deviennent à la fois des instruments à vent, au sens anal du terme - celui-là même que le bouffon d'Othello sous-entend dans la scène de l'aubade déjà évoquée : ces comemuses ${ }^{53}$ "parlent du nez", comme les humains frappés du mal napolitain, c'est-à-dire de la syphilis :

Clown. Why, masters, ha'your instruments been at Naples, that they speak $i$ the nose thus? ${ }^{54}$

Dans ce contexte anal et sexuel, la séquence "Naples" $\mu$ "windinstrument" $/$ "tail" entre en résonnance avec les clystères (clysterpipes ${ }^{55}$ ) qu'Iago, autre figure du fantastique infernal, voudrait voir entre les lèvres de Cassio:

Yet again, your fingers at your lips? would they were clyster-pipes for your sake. [Trumpets within] The Moor, I know his trumpet ${ }^{56}$.

Monde de l'inversion diabolique, l'enfer d'Othello voisine avec celui de Bosch, le traitement fantastique inversant les positions respectives du buccal et de l'anal, de l'instrument et du corps. Il est vrai que c'est sans doute l'instrument qui paie le plus lourd tribut aux similitudes déjà évoquées : sa constitution en peau de chèvre, la tête de chèvre dont il est souvent orné le prédestinent aux lectures diaboliques, puisque le diable, chacun le sait, sent le bouc. On sait également l'utilisation régulière qui est faite de la cornemuse dans la polémique et la caricature religieuses, par les 
catholiques comme par les réformateurs (illustration 3). Mais il a également des effets diurétiques reconnus, que l'on peut difficilement expliquer. Deux hypothèses peuvent cependant être avancées en l'absence d'une théorie définitive :

- lorsque Shylock fait allusion à cet "effet" particulier,

And others when the bagpipe sings $i^{\prime}$ th nose,

Cannot contain their urine ${ }^{57}$

l'incontinence semble devoir être prise dans le contexte comme synonyme d'émotion ${ }^{58}$, d'admiration incontrôlée, forme inverse du ravissement provoqué par les instruments à cordes. L'éthos dionysiaque/infernal de la cornemuse peut donc rendre compte de cet "effet" particulier; - d'autre part la loi des similitudes peut également suggérer une analogie au corps humain, non pas anale, mais urinaire: l'allusion très claire au pénis ("tail") et la ressemblance formelle entre la peau gonflée de la cornemuse et la vessie peuvent induire un effet pratique, si l'on suit la logique des "signatures" de Crollius, où l'isomorphisme est fonctionnel, où la ressemblance, une fois observée, est efficace : à mêmes formes, mêmes effets.

Aux vertus diurétiques on pourrait assez naturellement opposer la valeur nutritive du matériau musical, la notion de "pénétration" étant le truchement logique par lequel s'effectue la métaphore. L'analogie de la pénétration, réitérée dans l'immense majorité des traités théoriques sur la musique depuis le Timée de Platon, est le fondement même de la psychologie musicale à la Renaissance : le son musical, doué de souffle, de mouvement, de passion et de sens est assimilé à un "animé doué de raison" (a rational animal), capable de générer par l'émission d'une semence aérienne et d'agir sur le corps de ses auditeurs :

For [the song is] air, hot or warm, still breathing and somehow living; like an animal, it is composed of certain parts and limbs of its own and not only possesses motion and displays passion but even carries meaning like a mind, so that it can be said to be a kind of airy and rational animal. [It] ... generates offspring outside of itself by the emission of the seminal spirit ... so the vital $\&$ animal power, when it is most efficacious, not only acts powerfully on its own body when its spirit undergoes a very intense conception \& agitation through song but soon also moves a neighbouring body by emanation 59 . 
La pénétration du corps par l'élément musical ("song") est une idée reçue commode pour y greffer des interprétations littérales, alimentaires, architecturales ou sexuelles.

On connaît la boulimie musicale d'un Orsino ${ }^{60}$ qui veut rassasier son appétit amoureux par un excès de musique ("surfeit"), appliquant avant la lettre le concept d'homéopathie à la mélothérapie. On sait également que Jacques peut gober la mélancolie d'une chanson, comme une belette gobe des cufs ${ }^{61}$. Ces métamorphoses innocentes, on l'a vu, sont fondées sur la notion théorique de la "pénétration" du corps et de l'âme, par l'air mis en mouvement. L'ouïe est donc une fenêtre de l'âme, par laquelle le charme (carmen) donne l'assaut ${ }^{62}$ :

Heare you this soule-invading voyce, and count it but a voyce? ${ }^{63}$

Shakespeare inverse et complexifie l'image dans l'évocation du Carnaval vénitien: pour Shylock, nouvel Ulysse résistant aux assauts musicaux de sirènes chrétiennes, ce sont les oreilles de sa maison que sa fille doit fermer à la pollution sonore de la mascarade. Fenêtres et oreilles, pureté du foyer domestique et virginité, architecture et anatomie sont alors métaphoriquement associées dans un charivari verbal qui semble annoncer l'enlèvement de Jessica :

But stop my house's ears, I mean my casements,

Let not the sound of shallow fopp'ry enter

My sober house. ${ }^{64}$

C'est le même pouvoir de pénétration que Cloten recherche, non pas avec la musique festive du fifre, mais plus probablement avec celle plus grave - au double sens du terme - de la viole, associée à la voix d'enfant ("unpaved eunuch") :

I am advised to give her music a mornings, they say it will penetrate. [Enter musicians] Come on, tune : if you can penetrate her with your fingering, so: we'll try with tongue too: if none will do, let her remain [...] if this penetrate, I will consider your music the better : if it do not, it is a vice in her ears, which horsehairs and calves'-guts, nor the voice of unpaved eunuch to boot, can never amend ${ }^{65}$.

La musique se voit traitée en philtre amoureux. Sa posologie ("a mornings"), le mode d'administration expéditif ("come on, too, to 
boot"), le caractère systématique du traitement ("if none will do ... if it do not ..."), l'insistence assez lourde avec laquelle la musique et ses moyens instrumentaux (ou vocaux) sont réduits à leurs composantes animales organiques ("horse-hairs", "calves'guts") font de cette aubade un traitement officinal de bonne femme ("they say"), une recette érotique, dont le contrepoint pornographique assez évident ("penetrate her with your fingering", "we'll try with tongue too", "eunuch") évoque le scénario assez pervers de l'impuissance.

La référence ici est classique aux "effets" de telle ou telle musique en fonction de son "ethos" propre, de l'heure à laquelle telle planète est dominante, des qualités particulières de chaque instrument, de ses composantes, du mode choisi. Marsile Ficin y fait longuement allusion au chapitre 21 de son Iñe livre déjà cité :

Assuredly, [...] your spirit - by frequent use of Jovial, Mercurial, or Venereal harmony, a harmony performed while these planets are dignified - singing at the same time most intently and conforming itself to the harmony becomes Jovial, Mercurial, or Venereal ${ }^{66}$.

Ce "schème de culture", pour reprendre l'expression de Jean Jacquot ${ }^{67}$, fondé sur les correspondances intimes entre l'humain, le cosmique et linstrumental, rend ainsi compte des effets miraculeux ou prodigieux de l'harmonie musicale, qu'il s'agisse de l'extase ou de certains types de pouvoirs occultes. Dans le texte shakespearien, la ligne de sens principale s'inscrit dans cette idée reçue, "topos" de toutes les Apologies de la Musique, où passent comme dans un catalogue stéréotypé les incontournables figures du panthéon musical : Orphée, Amphion, Terpandre, le Roi David, Demodocus, Pythagore, etc. Il y a pourtant davantage: la ligne de sens secondaire, pornographique, dont l'intelligibilité est simultanée de la première, fait coïncider par superposition une image et un concept, en l'occurrence un signifiant, l'organe ("finger"; "tongue"), et un trope, l'instrument ("fingering" pour "technique instrumentale"; "tongue" pour "voix chantée"). La lecture simultanée des deux discours, véritable polyphonie sémantique, procède de l'anamorphose, vision double d'une même réalité picturale. Cette superposition de l'organe et de l'instrument est déjà inscrite dans la langue, pour peu qu'on la sollicite: Shakespeare ne s'en prive pas, qui enchaîne ou fond heart-strings (tendons du coeur) en harp-strings (cordes de harpe) ${ }^{68}$, wind-pipe (trachée) en organ-pipe (tuyau d'orgue $^{69}$ ), organ (organe) en organ (instrument ${ }^{70}$, frets (frettes) en 
frets (colère ${ }^{71}$ ). Aussi la voix humaine, modèle de toutes ces "voix artificielles" (artificial voyces) que sont les instruments de musique, connait-elle les avatars de la trompette ${ }^{72}$ ou du tambour $^{73}$, lorsqu'elle est guerrière, de l'orgue ${ }^{74}$ quand elle est prophétique ou inspirée, de la flûte ${ }^{75}$, lorsqu'elle tente, malgré son format minime de dire de grandes vérités : la flûte par laquelle Hamlet se définit à Guildenstern a pour ethos réputé celui de la douceur et de l'intimité; c'est la flûte d'Angleterre, ou flûte douce, réputée facile à jouer, d'une pureté de timbre quasi-parfaite connotant régulièrement, et jusqu'à nos jours, l'innocence et souvent $l^{\text {'enfance }}{ }^{76}$. Tout en donnant de lui-même une représentation éthique et symbolique, fondée sur les connotations d'un instrument connu et populaire, Hamlet énonce l'argument central de son paradoxe "little organ" - "excellent voice" («à petit instrument, grande voix»); Hamlet fait ainsi un commentaire cryptique mais perceptible sur les jeux de l'apparence, du mensonge et de la vérité, alors même qu'il s'apprête à pratiquer lui aussi l'hypocrisie (il va effectivement "jouer la comédie" à sa mère et nous y prépare explicitement à la fin de la scène), mais il trame également en double (voire triple) enterdre une série d'allusions perverses à la chasse et au corps : prenant Guildenstern à part, il se pose en gibier qu'on voudrait prendre au filet

why do you go about to recover the wind of me, as if you would drive me into a toil? ${ }^{77}$

C'est probablement, comme pour le bouffon d' Othello, sur l'équivocité de "wind" que s'articule le discours fantastique d'Hamlet : la métamorphose cynégétique ("prendre le vent") induit, par association, le discours allusif sur la flûte ("instrument à vent") que les comédiens viennent de présenter sur scène. De gibier, Hamlet devient instrument. L'enchaînement des références corporelles, l'insistence marquée sur les trous ("ventages", "stops") et la nécessité de les boucher donnent une séquence dont l'analité est une fois de plus corollaire de la dimension pneumatique :

Govern these ventages ... give it breath with your mouth ... these are the stops ... how unworthy a thing you make of me ... you would play upon me ... you would seem to know my stops ... this little organ ...78. 
La réification indigne qu'Hamlet propose de lui-même de manière provocante dans son autoportrait, cette vision d'un corps complètement bouché, dont les trous seraient étanches, font penser au masochiste qui, selon Deleuze, «se fait coudre [...] pour que tout soit scellé bien clos»79. Mais l'anamorphose scénique dépasse le cadre des analogies formelles ou éthiques, puisqu'en tendant la flûte à Guildenstern et en s'identifiant à elle, Hamlet non seulement rejoue une version désacralisée de l'eucharistie («Prenez, ceci est mon corps ...»), mais il se pose aussi en tant que simple instrument (organ), persona exigeant de son auteur le souffle compétent du dramaturge qui saura lui donner voix («make it speak»). Ce commentaire métadramatique s'inscrit déjà dans une scène de thêâtre dans le théâtre au sens le plus conventionnel, où Hamlet se sert d'un accessoire scénique ("stage property"), la flûte, comme d'un miroir ou d'un totem. L'autoportrait métaphorique, à la manière d'un "portrait chinois", sert donc de prétexte à une exploration symbolique des structures mêmes de la dramaturgie et de la représentation.

L'instrumentarium animé de Shakespeare comprend bien d'autres figures hybrides où l'humain s'anastomose à l'instrument : c'est par exemple Mowbray dont l'exil linguistique s'exprime sous la forme d'une viole sans cordes («a stringless instrument»), ou la fille d'Antioche dont Péricles décrypte le mystère incestueux en cryptant son propre message :

You are a fair viol, and your senses the strings,

Who, fingered to make men his lawful music,

Would draw heaven down and the gods to hearken;

But being played before your time,

Hell only danceth at so harsh a chime. ${ }^{80}$

L'analogie évidente entre rapport sexuel ordonné et musique harmonieuse se prolonge en un conceit où le corps de la femme est l'instrument dont l'homme doit jouer sans contre-temps. La folie de Lear comme celle d'Hamlet reçoivent pour définition et remède l'expression antiphrastique de l'ordre musical («sweet bell out of tune / senses ... untuned ${ }^{81}$. Coriolan pour gagner les voix électorales de la plèbe, se refuse à changer sa voix de guerre («throat of war» ${ }^{82}$ ) en une voix de femme ou de castrat («eunuch»). En suggérant l'hypocrisie du politique, il ne s'affirme pas moins comme machine à tuer, à l'unisson avec les instruments de guerre («choiring with my drum»), qui se moque de toutes les forces du 
renouveau et de la vie symbolisées dans la fragile image de la nourrice chantant une berceuse à des bébés. Il y aurait beaucoup à dire sur le motif de la castration : elle paraît évidente dans eunuch, à cela près que l'image du castrat se complexifie d'une anamorphose instrumentale sous la forme d'un artefact: l'eunuque était en effet un instrument de la Renaissance, fort bien décrit par le Père Mersenne ${ }^{83}$, et dont la caractéristique essentielle est qu'il émet, par son anche dissimulée, un timbre doux analogue à celui d'une flûte. Même si ce sens de "eunuch" n'est pas attesté par l'O.E.D., et même si le contexte rend évidente l'équation "eunuch" = "castrat", il est néanmoins possible de proposer, comme en image subliminale, une vision instrumentale cohérente, pour peu que l'on prenne les signifiants "drum" - "pipe" - "small" au sens littéral, c'est-à-dire instrumental.

L'instrument apparaît dans l'ensemble de ces métamorphoses verbales comme l'artefact le plus évocateur du corps unifié d'avant la désintégration. Même si (et peut-être parce que) le traitement de cette intégrité se fait le plus souvent sur le mode antiphrastique du désordre et de la dissonance, il porte par nature la nostalgie d'une unité perdue. La vision médiévale de l'homme-instrument subit sur la scène shakespearienne d'innombrables avatars, l'hybridation y étant présentée soit sur le mode symbolique, soit sur celui de la dérision. Les topoi sur la musique, littéralisés à des fins dramaturgiques complexes, ou énoncés sous forme de métaphore poétique, sont des éléments authentiques d'une poétique autant que d'une pratique dramaturgique.

Pierre ISELIN

Université de Limoges

\section{NOTES}

1. The Tatler, $\mathrm{N}^{\circ} 153$, Saturday, April 1, 1710.

2. As You Like It, II.vii.161-3.

3. Prologue de Pantagruel : Rabelais. L'Intégrale, G. Demerson, ed., Paris, Seuil, 1973, p. 215.

4. Instrument du type de clavecin/clavicorde. 
5. Le Quart Livre, Chap. XXXI, Ibid., pp. 667-668. On ne saurait ici adhérer ni à la traduction de "jeu de réguales" en "jeu de flûtes" ni à celle de "cornet à bouquin" en "trompe de chasse", proposées par l'éditeur.

6. Hollander, The Untuning of the Sky. Ideas of Music in English Poetry, 1500-1700, Princeton University Press, 1961, p. 159.

7. Joscelyn Godwin, Music, Mysticism and Magic. A Sourcebook, London, Routledge \& Kegan Paul, 1986; Hollander, op. cit.; Gretchen Ludke Finney, Musical Backgrounds for English Literature: 1580-1650, Westport, Conn., Greenwood Press Publishers, 1962, rep. 1976; James Hutton, "Some English Poems in Praise of Music" in English Miscellany, M. Praz, ed. Rome, 1951, vol., pp. 1-63.

8. Lomazzo, Trattato dell'arte della pittura, 1584; cité par R. Wittkower, Architectural Principles in the Age of Humanism, 3rd edition, London, 1962, p. 118.

9. En particulier les cathédrales de Valladolid, Ségovie, Salamanque; voir Wittkower, op. cit., pp. 119-120.

10. Le plus souvent identifié à John Case, l'auteur d'une Apologie de la musique (Apologia Musices), Publiée à Oxford, chez le même Barnes en 1588.

11. Première occurrence attestée par O.E.D.

12. The Praise of Musicke, Bames, ed., Oxford, 1586, chap. 1. "The antiquitie and originall of musicke", p. 2, texte dont je prépare actuellement une édition critique. 106.

13. Charles Butler, The Principles of Musicke, London, 1636, p.

14. Voir Godwin, op. cit., chap. 27, "Marsilio Ficino", pp. 117122, citant De Vita Coelitus Comparanda, livre III, chap. 21; "Henri Cornelius Agrippa", chap. 29, pp. 127-132, citant Three Books of Occult Philosophy, livre I, chap. 69-71, livre II, chap. 25; "Robert Fludd", chap. 33, pp. 143-147, citant le Tractatus Apologeticus Integritatem Societatis de Rosea Crucis Defendens, chap. 6; "Athanasius Kircher", chap. 35, pp. 153-161, citant un extrait de Musurgia Universalis, vol. III.

15. Propaedeumata Aphoristica, Aphorism XI, London, 1618; cité par G. Finney, op. cit., p. 2.

16. "The powers above do tune the harmony of this peace", Cymbeline, V.v.467.

17. Robert Fludd, Utriusque Cosmi ... Historia, Oppenheim, 1617. Tract. I, lib. III, cap. III, p. 90; discuté par Marin Mersenne, traité de l'Harmonie Universelle, Paris, 1627, livre II, Théorème V, pp. 443 et sqq. 
18. Athanasius Kircher, Musurgia Universalis, Rome, 1650, rep. Hildesheim, Holms, 1970, vol. II, p. 366, Iconismus XXII, Harmonia Primi Diei.

19. The Tempest, III.iii.98.

20. Pour ce rapport entre air, son musical, et physiologie de la perception, voir infra, en particulier note 59.

21. D. A. Alciati, Emblemata, Lyon, 1551, sig. A8v.

Difficile est, nisi docto homini, tot tendere chordas,

Unaque; si fuerit ron bene tenta fides.

Ruptave (quod facile est) perit omnis gratia conchae.

Illeque praecellens cantus, ineptus erit.

cf. la traduction proposée par l'auteur dans l'édition fraçaise de Toutes les Emblèmes de M. André Alciat, Lyon, 1558, réédité par les Amateurs de Livres, Bibliothèque Interuniversitaire de Lille ${ }^{2}$ 1989, p. 30 :

Difficile est tant de chordes estendre

Fors qu'au prudent. Si l'une ne veult tendre,

Ou rompue est (ce qu'est facilement)

Grace du son se perd totallement.

Voir aussi, pour l'inage du Roi-Musicien réparant un luth brisé : Geoffrey Whitney, A Choice of Emblems, Leyden, 1586, Sig. M2v.

22. On peut opérer le même rapprochement pour son diminutif fidiculae, désignant la petite lyre, mais également le chevalet, instrument de torture oì de gros boulets étiraient les jambes.

23. "De même que le corps sombre dans la paralysie et l'infirmité des membres lorsque les nerfs se rompent, de même la cité par ses membres dissonants sombre dars l'horreur et la confusion au risque de ces mêmes citoyens" (John Case, Apologia Musices, Oxford, 1588, p. 50). [Une édition en langue nodeme de ce texte est actuellement en préparation.]

24. Richard III, I.i.18.

25. Richard III, I.i.24-27.

26. Othello, III.i.15-17.

27. Gosson, The Schoole of Abuse (1579), re-ed. Edward Arben, Birmingham, 1868, pp. 25-26.

28. Voir Love's Labours Lost, V.ii.603-605.

29. Marston, The Malcontent, Induction, 17-20.

30. artificial voyce désigne en fait l'instrument de musique, l'artifice par lequel est imitée la voix humaine.

31. Tottel, Songs and Sonets, 1557.

32. The Merchant of Venice, II.v.30.

33. Plutarch, trad. North (1579): Life of Alcibiades, from Lives, Englished by Thomas North; repr. Tudor Translations, London, 1895, VII. 91-92.

34. Voir note 21. 
35. The Praise of Musicke, chap. I, p. 16 : "... Linus. Whose ungracious scholar Hercules being controlled by him for his rawnes made such untoward Musike betwixt his Maisters harpe and his heade, that he beate out the sides of the one and the braines of the other."

36. The Taming of the Shrew, II.i.147-156.

37. En témoigne le passage suivant, de tradition homérique, extrait de The Praise of Musicke (p. 57):

Touching the first effects of music we read that Agamemnon going to the war of Troy left behind him Demodocus, an excellent musician, skilfull in Modo Dorio, to keep chast his wife Clitemnestra, whom he nicely had in suspicion of wantonnes and levity with Aegistus.

Whereupon it is recorded that as long as Demodocus lived, Clytemnestra remayned faithfull to her husband: but when Aegistus, for that purpose had murthered him, shee gave over her selfe to satisfie his adulterous appetite.

38. Emanuel Winternitz, Musical Instruments and their Symbolism in Western Art, Yale University Pres, 1967, rep. 1967, p. 157 , note 1 .

39. Voir Ovide, Métamorphoses, I, 689 sqq.

40. The Praise of Musicke, p. 13, citant Polydore Virgile.

41. William Drummond, Sonnet VII :

To his lute.

When Orpheus strook th'inspired Lute,

The Trees danc'd round, and understood

By sympathy the voice of the Wood.

(cf. Boèce, Consolation, III, XII :

Vates Thercius [...]

Postquam flebilibus modis

Silvas currere mobiles

Amnes stare coegerat $[\ldots]$

Illic blanda sonantibus

Chordis carmina temperans [...])

42. Pierre Trichet, Traité des Instruments de musique (ca. 1640). Edition moderne, Société de musique d'autrefois, Neuilly sur Seine, 1957.

43. Cor. Agrippa (trad. J.F.) : «No man can make the roaring of Linos, the braying of Asses, the grunting of Hogs to be harmonious : neither can the strings made of Sheeps and Wolves guts, be brought to any agreement, because their foundations are dissonant ...» (cité par Godwin, op. cit., p. 132).

44. Two Gentlemen of Verona, III.ii.77-80.

45. Michel du Pressoir (d. 1302) : Jésus Christ «a desséché la corde de sa chair par le jeûne, et sur la croix, il l'a tendue, comme la corde d'un instrument est tendue entre deux morceaux de bois». Cité par 
Roger Cotie, Mhsique et Symbolisme, Résonances Cosmiques des Instruments et des Euvres, Saint Jean de Braye, Editions Dangles, 1988, p. 60 .

46. Voir Roger J. V. Cotte, op. cis., p. 65.

47. Oswald Crollius, traité des Signstures, ou Vraye ef Vive Anatomie dit Grand st Peti Monde, traduction française, Lyon, 1624. (Repr. Collection Sebastiani, 1976).

48. Much Ado about Nothing, II.iii.58-60.

49. Platon, Le Timée, $47 \mathrm{c}$ et sqq.

50. Macrobius, Commentary on the Dream of Scipio, book II, chap. 1, 2, 3, trad. Stahl, New York, 1952, pp. 185-197.

51. Richard Hooker, The Laws of Ecclesiastical Polity, bk. V, sec. XXXVII, ed. Ronald Bayne, London \& New-York, 1925, II, p. 146: "There is also that carrieth as it were into ecstasies, filling the mind with an heavenly joy and for the time in a manner severing it from the body."

52. Ronsard, A Madame Marguerite, Le Cinquième Livre des Odes, Cuvres Complètes, ed. Faul Laumonier, Paris, 1914-18, II, p. $379:$

Avecque ta voix nompareille

Leur tires l'âme par l'oreille

D'un vertueux enchantement.

voir aussi l'Ode au Prince de Melphe, Pause X, Poésies françaises et latines de Joaquim du Bellay, ed. E. Courbet, Paris, 1919, II, 95 :

Là d'une musique foumie

Nous avions la doulce harmonie,

Dont les discours mélodieux

De mile douceurs nompareilles

Tirent l'âme par les oreilles,

Nous ferons compaignons des dieux.

53. Voir Pierre Iselin, "Les Musiques d'Othello", in Autour d'Othello, Amiens, Presses Universitaires de Picardie, 1987 [Sterne]. p. 68 .

54. Othello, Mi.i.3-4.

55. Voir Pierre Iselin, op. cit., p. 66; Rosalind King établit un rapprochement encore plus direct entre 'clyster-pipes' et 'pipes' dans 'Then Murther's out of Tune', The Music and Structure of Othello, Shakespeare Survey no 39 , pp. 149-158.

56. Othello, II.i.175-8.

57. The Merchant of Verice, IV.i.49-50.

58. The Winter's Tale, IV.iv.185.

59. Marsilio Ficino, De Vita, libri tres, trad. Carol Kaske, Mediaeval \& Renaissance Texts, Binghampton, New-York, 1986, cité par Godwin, op. cir., pp. 120-121.

60. Twelfth Night, I.i.1 et sqq. 
61. «I can suck melancholy out of a song, as a weasel sucks eggs.» (As You Like It, II.v.11-12) musics.»).

62. "assail" (Cymbeline, II.iii.38: «I have assail'd her with

63. Sidney, Apostrophel and Stella, Seventh Song, M. Poirier, ed., Paris, Aubier, 1962, p. 152.

64. The Merchant of Venice, II.v.34-36.

65. Cymbeline, II.iii.11-29.

66. Marsilio Ficino, op. cit., p. 122.

67. Jean Jacquot, "L'éloge de la musique, grandeur et décadence d'un schème de culture", Revue Belge de musicologie, XX (1-4), 1966, pp. 91-110.

68. Richard III, IV.iv.364-365 et Henry VIII, III.ii.105-106.

69. King John, V.vii.21-24, et par dislocation de "organ-pipe" en "pipe ... organ" : Twelfth Night, I.iv.32-33.

70. Twelfth Night, I.iv.32-33 et Hamlet III.ii.359.

71. The Taming of the Shrew, II.i.152; Hamlet, III.ii.362.

72. Coriolan, I.i.116: «The tongue our trumpeter»; "to trumpet» (Othello, I.iii.248-50 et Pericles, I.i.146).

73. Coriolan, III.ii.112-115:

My throat of war be turn'd,

Which choir'd with my drum, into a pipe

Small as an eunuch, or the virgin voice

That babies lull asleep!

74. Twelfth Night, I.iv.32-34; All's Well that Ends Well, II.i.176; Much Ado about Nothing, IV.i.266; The Tempost, III.iii.98; King John, V.vii.23.

75. Hamlet, III.ii.337-338.

76. «Indeed he hath played on this prologue like a child on a recorder; a sound, but not in government" (A Midsummer Night's Dream, V.i.122-123).

77. Hamlet, III.ii.337-338.

78. Ibid.

79. G. Deleuze, Mille Plateaux, Paris, 1983, pp. 186-87.

80. Pericles, I.i.82-6.

81. King Lear, IV.vii.15-17.

82. Voir note $\mathrm{n}^{\circ} 73$.

83. Marin Mersenne, op. cit. : «Le quatrième ["= Chalumeau ou Fluste"] est appelé Eunuch par quelques-uns, [...] elle ne fait point d'autre son que celui de la bouche, ou de la langue qui parle, dont elle augmente la force \& la résonance par le moyen de sa longueur \& de sa capacité, \& par une petite peau de cuir mince, \& déliée comme la peau d'un oignon, dont on affuble le haut, où l'on void $A$, afin que le vent et la voix que l'on pousse par le trou $B$, qui fait l'emboucheure, aille frapper cette peau comme un petit tambour, qui donne un nouvel 
agrément à la voix par ses petits tremblements qui la réfléchissent [...]. Or l'on fait quatre ou cinq parties différentes de ces Flustes pour un concert entier, qui a cela par dessus toutes les aurres Flustes, qu'il imite davantage le concert des voix, car il ne luy manque que la seule prononciation, dont on approche de bien près avec des Flustes.» 